\title{
Pharmacological induction of leukotriene B4-12- hydroxydehydrogenase suppresses the oncogenic transformation of human hepatoma HepG2 cells
}

\author{
LAI WEI $^{1}$, JIE LIU ${ }^{2}$, X. CHRIS LE ${ }^{2}$, YIFAN HAN ${ }^{3}$, YAO TONG ${ }^{1}$, ALLAN S.Y. LAU ${ }^{4}$ and JIANHUI RONG ${ }^{1}$ \\ ${ }^{1}$ School of Chinese Medicine, Li Ka Shing Faculty of Medicine, The University of Hong Kong, \\ 10 Sassoon Road, Pokfulam, Hong Kong, SAR, P.R. China; ${ }^{2}$ Department of Laboratory Medicine and \\ Pathology, Faculty of Medicine and Dentistry, 10-102 Clinical Sciences Building, University of Alberta, \\ Edmonton, Alberta T6G 2G3, Canada; ${ }^{3}$ Department of Applied Biology and Chemical Technology, Hong \\ Kong Polytechnic University, Hung Hom, Kowloon; ${ }^{4}$ Molecular Chinese Medicine Lab, Li Ka Shing Faculty \\ of Medicine, The University of Hong Kong, 10 Sassoon Road, Pokfulam, Hong Kong, SAR, P.R. China
}

Received February 28, 2011; Accepted April 15, 2011

DOI: 10.3892/ijo.2011.1082

\begin{abstract}
Leukotriene B4-12-hydroxydehydrogenase(LTB4DH) is characterized as a chemopreventive and tumor suppressor gene. The aim of this study was to investigate the pharmacological induction of LTB4DH and potential anticancer activity. Using HepG2 cells as a cellular detector, we successfully isolated the active compounds from the herbs Radix Astragali and Radix Paeoniae Rubra through a bioactivity-guided fractionation procedure. Using various analytical techniques including electronic spray ionization-mass spectrometry (ESIMS) and nuclear magnetic resonance (NMR), gallic acid (GA) was identified as the active compound from Radix Paeoniae Rubra whereas the active compound from Radix Astragali, designated as RA-C, was also purified to the extent that it is now suitable for further identification. We found that the active compounds from these two different herbs synergistically induced LTB4DH expression in a dose- and time-dependent manner. A key finding was that commercial GA in combination with purified RA-C attenuated the focus formation and anchorage-independent growth, two indexes of in vitro oncogenic transformation, of HepG2 cells via the induction of LTB4DH expression. Moreover, the combination of GA and purified RA-C significantly induced G2/M cell cycle arrest in HepG2 cells. Our results demonstrated for the first time that GA and purified RA-C suppress the in vitro
\end{abstract}

Correspondence to: Dr Jianhui Rong, School of Chinese Medicine, Li Ka Shing Faculty of Medicine, The University of Hong Kong, 10 Sassoon Road, Pokfulam, Hong Kong, SAR, P.R. China E-mail: jrong@hkucc.hku.hk

Key words: chemoprevention, leukotriene B4-12-hydroxydehydrogenase, leukotriene B4, oncogenic transformation, cell cycle arrest, gallic acid, Radix Astragali, Radix Paeoniae Rubra, bioactivityguided fractionation oncogenic transformation of HepG2 cells via the induction of LTB4DH expression. Importantly, pharmacological induction of LTB4DH represents a potential alternative strategy for the therapy of hepatocellular carcinoma.

\section{Introduction}

Leukotriene B4 (LTB4) is a potent inflammatory mediator generated from the cell membrane arachidonic acids by 5-lipoxygenase (5-LOX) pathway $(1,2)$. LTB4 is well-known for its role in the pathogenesis of various inflammatory diseases, such as asthma, psoriasis, rheumatoid arthritis, inflammatory bowel disease and cancers (3). The production of LTB4 is strongly enhanced in human cancer cells, for example, LTB4 level was increased by up to 10-30-fold in oral squamous cell cancers (4). Such aberrant elevation of LTB4 level in cancer cells is a result of the overexpression of LTB4 biosynthetic enzymes, such as 5-LOX and leukotriene A4 hydrolase $(5,6)$. LTB4 stimulated the proliferation of colon cancer cell lines HT-29 and HCT-15 in a time- and concentrationdependent manner (7). By activating its receptor, LTB4 could antagonize glucocorticoid-induced inhibition of lymphoma growth and the inhibitory effect of nordihydroguaiaretic acid (NDGA) on breast cancer cell line MDA-MB-231 $(3,8)$. Under the physiological conditions, biologically active LTB4 is metabolized via sequential $\omega$-oxidation and $\beta$-oxidation (2). This metabolic pathway serves as an effective control of the excessive/residual LTB4. Thus, the control of LTB4 formation is a potential target for pharmacological intervention of various cancers (9).

Leukotriene B4-12-hydroxydehydrogenase (LTB4DH) is a multifunctional enzyme capable of catalyzing the oxidation of LTB4, the reduction of 15-oxo-prostaglandins (15-PGs), and the inactivation of 15-oxo-PGE and lipoxin A4 (10). In addition, LTB4DH was recently found to activate anticancer pro-drugs as well as to detoxify exogenous/endogenous cytotoxic compounds (11). LTB4DH is not constitutively 
expressed in most of cell types. Once activated, LTB4DH catalyzes the conversion of cellular LTB4 to less active 12-oxo-LTB4, providing an alternative control of LTB4mediated proinflammatory signals. Various conjugated diene metabolites of LTB4 were detected in human urine (12). Compared with the physiological metabolic pathway, LTB4DH is of importance in the control of LTB4 and/or 15-PGs receptor-mediated signals $(10,13)$. Loss of LTBDH expression either by genetic or epigenetic mechanism resulted in elevated LTB4 level in tumors, supporting the growth of cancer cells in a similar fashion to the role of 15-hydroxyprostaglandin dehydrogenase (15-PGDH) in lung and other cancers (14-16). In contrast, ectopic expression of LTB4DH could effectively attenuate the stimulatory effects of LTB4 on breast cancer cells (17). Thus, pharmacological induction of LTB4DH expression by novel reagents may be a new anticancer mechanism. Of note, LTB4DH was recently isolated from the rat liver as a dithioethione-inducible gene 1 (DIG-1) (18). The induction of LTB4DH may largely contribute to the chemopreventive activity of dithiolethiones.

Herbal medicines are an important resource of botanical compounds that possess anticancer and chemopreventive properties against various cancers (19-21). For example, Radix Paeoniae Rubrae and Radix Astragali are often prescribed as anticancer therapy in traditional Chinese medicine. Radix Paeoniae Rubrae, the root of plant Paeonia lactiflora Pall (Family Ranunculaceae), is used to stimulate blood circulation, remove blood stasis, clear heat, cool blood, remove stagnant blood and minimize swelling (22). A number of monoterpene glycosides, galloyl glucoses and phenolic compounds have been isolated as bioactive components (23). In particular, gallic acid (GA), galloyl derivatives and paeoniflorin are important anti-inflammatory and anticancer components. On the other hand, Radix Astragali, the dried root of Astragalus membranaceus (Fisch.) Bunge and Astragalus mongholicus Bunge (Fabaceae) is well-known as an immunostimulant, adaptogenic, hepatoprotective, diuretic, antidiabetic, analgesic, expectorant, and sedative (24,25). Radix Astragali aqueous extract was recently found to be antimutagenic and chemopreventative (26). The active compounds of Radix Astragali are principally saponins and polysaccharides. The anticancer activity of Radix Astragali is mediated through immunological mechanisms as reviewed (27).

It is noteworthy that different herbal compounds in the formulations may interact with each other to exhibit novel activities, which can not be explained by individual ingredients. Partly for this reason, we recently described a genome-wide biological response fingerprinting (BioReF) approach to study the biological activity of complex botanical formulation (28). Our strategy uses human cells as the cellular detector to monitor the changes of mRNA expression in response to the botanical formulations by genome-targeting DNA microarrays. As a result, we found that LTB4DH was greatly induced by a formulation composed of Radix Astragali and Radix Paeoniae Rubra in hepatocellular carcinoma cell line HepG2. These results prompted us to further characterize the active compounds from these herbs and the molecular mechanisms underlying the induction of LTB4DH.

The objective of the present study was to investigate the pharmacological induction of LTB4DH and explore the anticancer activity of isolated LTB4DH inducers. We firstly developed a bioactivity-guided fractionation procedure for rapid isolation of the active compounds from the herbs Radix Astragali and Radix Paeoniae Rubra. The active compounds were identified by various analytical techniques including electronic spray ionization-mass spectrometry (ESI-MS) and nuclear magnetic resonance (NMR) spectroscopy. We subsequently examined the effects of the active compounds on LTB4DH expression and the focus formation and anchorage-independent growth, two indexes of in vitro oncogenic transformation, of HepG 2 cells. We anticipated that pharmacological induction of LTB4DH would serve as a potential alternative strategy for the therapy of hepatocellular carcinoma.

\section{Materials and methods}

Cell culture and reagents. Human hepatocellular carcinoma cell line HepG2 was obtained from American Type Culture Collection (Rockville, MD, USA) and cultured in Eagle's minimum essential medium (MEM) containing 10\% fetal bovine serum and supplemented with $100 \mathrm{U} / \mathrm{ml}$ penicillin and $100 \mu \mathrm{g} / \mathrm{ml}$ streptomycin. The cells were maintained in a humidified incubator under $5 \% \mathrm{CO}_{2}$ at $37^{\circ} \mathrm{C}$. The dried aqueous Radix Paeoniae Rubra and Radix Astragali extracts were purchased from a local pharmaceutical company Nong's Company, Hong Kong, China. LTB4DH antibody was purchased from Santa Cruz Biotechnology (Santa Cruz, CA, USA). The antibodies against $\beta$-actin and rabbit $\operatorname{IgG}$ were purchased from Sigma-Aldrich (St. Louis, MO, USA). The oligonucleotide primers specific for LTB4DH and $\beta$-actin were purchased from Genome Research Centre, University of Hong Kong. Other chemicals were obtained from SigmaAldrich Co. unless indicated otherwise.

Measurement of cell viability. Cell viability was evaluated by a standard colorimetric assay for the reduction of 3-[4,5-dimethylthiazol-2-yl]-2,5-diphenyltetrazolium bromide (MTT) catalyzed by mitochondrial reductase (28). Briefly, at the end of drug treatment, the cell monolayer was incubated in MTT solution $(0.5 \mathrm{mg} / \mathrm{ml})$ in phosphate-buffered saline (PBS) for $4 \mathrm{~h}$. The formation of purple formazan was quantified by measuring the absorbance at $570 \mathrm{~nm}$ on a microplate reader (Bio-Rad, USA).

Apparatus and instruments. The HPLC system consisted of two Waters 626 Model LC pumps, a Waters 717 Plus autosample injector, a Waters 996 Model photodiode array detector (DAD), a Waters 600S model system controller and a gradient generator. All samples were analyzed on an Alltima ${ }^{\mathrm{TM}} \mathrm{HP}$ C18 column (Alltech, USA, $5 \mu \mathrm{m}, 250 \times 4.6 \mathrm{~mm}$ i.d.) coupled to an AllGuard ${ }^{\mathrm{TM}}$ guard column (Alltech, $7.5 \times 4.6 \mathrm{~mm}$ i.d.). UV absorption was measured with a spectrum ranging from 200 to $400 \mathrm{~nm}$. For the fractionation of Radix Paeoniae Rubra extract, a binary gradient elution system was made using acetonitrile as solvent $\mathrm{A}$ and water as solvent $\mathrm{B}$. The gradient elution was performed as follows: $0-5 \mathrm{~min}, 10-15 \% \mathrm{~A}$; 5-25 min, 15-22\% A; 25-45 min, 22-70\% A; 45-46 min, $70-80 \% \mathrm{~A} ; 46-50 \mathrm{~min}, 80 \% \mathrm{~A}$. The flow rate of mobile phase was $0.8 \mathrm{ml} / \mathrm{min}$, and column temperature was maintained at 
$25^{\circ} \mathrm{C}$. The PDA detector was set at $230 \mathrm{~nm}$, and the on-line UV spectra were recorded in the range of 195-400 $\mathrm{nm}$. For the fractionation of Radix Astragali extract, acetonitrile (A) and $\mathrm{H}_{2} \mathrm{O}(\mathrm{B})$ were used to generate a gradient mobile phase. The samples were eluted by $5 \%$ A for the first $10 \mathrm{~min}$, a linear gradient to $80 \% \mathrm{~A}$ at $40 \mathrm{~min}$ and subsequent gradient to $95 \%$ $\mathrm{A}$ at $45 \mathrm{~min}$, and finally $100 \% \mathrm{~B}$ for another $10 \mathrm{~min}$. The flow rate was set at $1.0 \mathrm{ml} / \mathrm{min}$.

Bioactivity-guided fractionation of Radix Paeoniae Rubra and Radix Astragali extracts. For Radix Paeoniae Rubra, the dried aqueous extract (200 grams) was suspended in 1 liter of Millipore water and heated at $80^{\circ} \mathrm{C}$ for $45 \mathrm{~min}$. Following the centrifugation at $4000 \mathrm{rpm}$ for $30 \mathrm{~min}$, the supernatant was recovered and sequentially precipitated with 3 volumes of $100 \%$ ethanol. The soluble materials were dried using a rotary evaporator (Büchi, Germany) under vacuum. The dried residues were dissolved in Millipore water and assayed for the ability to induce LTB4DH expression in HepG2 cells. The fraction which significantly increased the expression of LTB4DH was further separated by semi-preparative HPLC. Thirteen fractions were generated and subjected to the determination of LTB4DH induction. Finally, the active fraction with a single peak in HPLC profile was analyed by LC/MS and NMR. It should be noted that the fractions generated from Radix Paeoniae Rubra extract must be assayed in the combination with Radix Astragali extract. To isolate the active compound from Radix Astragali extract, designated as RA-C, the fractionation was started with $2 \mathrm{~kg}$ of the dried aqueous extract and processed in a similar fashion as described above. The fractions isolated from Radix Astragali were assayed similarly in the combination with Radix Paeoniae Rubra extract.

Chemical identification by mass spectrometry and ${ }^{1} \mathrm{H} N M R$ spectroscopy. The purified active compounds were analyzed on an LC-MS/MS system consisting of an API 2000 Qtrap hydrid QqQ-linear ion-trap mass spectrometer equipped with TurboIonSpray $^{\mathrm{TM}}$ source (Applied Biosystems, Foster City, CA, USA) and HP 1100 series HPLC system (Agilent Technologies, Palo Alto, CA, USA) equipped with a XTerra ${ }^{\mathrm{TM}}$ Ms C18 column (3.5 $\mu \mathrm{m}, 2.1 \times 150 \mathrm{~mm}$; Waters, Milford, MA, USA). HPLC fractions were monitored by MS while the scan range was 200-1000 amu. Data acquisition was interfaced to a computer workstation running Analyst1 1.4.1 (Applied Biosystems). ${ }^{1} \mathrm{H}$ NMR spectra were recorded on a JOEL JNM-GX400 NMR spectrometry.

Reverse transcription polymerase chain reaction (RT-PCR) detection. Following the treatment with herbal drugs such as Radix Paeoniae Rubra and Radix Astragali extracts, the total RNAs were isolated using RNeasy mini kit (Qiagen, USA) and converted into corresponding cDNAs using SuperScript III reverse transcriptase and random hexamer primers (Invitrogen, Carlsbad, CA, USA). LTB4DH mRNA (NM_012212) was detected using specific primers: sense, 5'-GAGCTTCAGGATGGTTCGTA-3'; antisense, 5'-TCA TGCTTTCACTA TTGTCTTCC-3', whereas $\beta$-actin mRNA (NM_001101) as internal control was detected using specific primers: sense, 5'-GGCACCACACCTTCTACAATGA-3'; antisense, 5'-GGAGTTGAAGGTAGTTTCGTGGA-3', PCR amplifications were performed as follows: after an initial denaturation at $94^{\circ} \mathrm{C}$ for $3 \mathrm{~min}, 35$ cycles of $94^{\circ} \mathrm{C}$ for $30 \mathrm{sec}$, $57^{\circ} \mathrm{C}$ for $30 \mathrm{sec}$, and $72^{\circ} \mathrm{C}$ for $30 \mathrm{sec}$, extension at $72^{\circ} \mathrm{C}$ for $10 \mathrm{~min}$. PCR products were analyzed by gel electrophoresis in $1.2 \%$ agarose containing ethidium bromide and visualized under UV light.

Generation of HepG2 cell lines stably carrying LTB4DH cDNA and shRNAi. Full length LTB4DH cDNA (NM_012212) was cloned from HepG2 cells using specific primers sense 5'-GATCGAATTCGAGCTTCAGGATGGTTCGTA-3' and antisense 5'-GATCCTCGAGTCATGCTTTCACTATTGTC TTCC-3' by reverse transcription-polymerase chain reaction using the Accume RT-PCR system kit (Invitrogen). PCR product was cut by EcoRI and XhoI and inserted into pcDNA3.1 expression vector (Invitrogen), accordingly. Several clones bearing LTB4DH cDNA inserts were isolated and fully sequenced at Tech Dragon Ltd. (Hong Kong). Expression vector pRS-shRNA bearing LTB4DH specific shRNAi and control shRNAi sequences were purchased from OriGene Technologies (Rockville, MD, USA). Transfection of HepG2 cells with pcDNA3.0 vector, LTB4DH cDNA, LTB4DH specific shRNAi or control shRNAi construct was carried out by using FuGene 6 transfection reagent (Roche, USA). Stable clones were selected in the presence of G418 $(1 \mathrm{mg} / \mathrm{ml})$ over a period of two weeks. Selected clones were evaluated by RT-PCR for LTB4DH expression or the presence of shRNAi sequences.

Western blot analysis. Following the drug treatment, the cellular proteins were extracted and analyzed for protein expression as previously described $(29,30)$. Briefly, $30 \mu \mathrm{g}$ of the cellular proteins were resolved by electrophoresis in $10 \%$ SDS-polyacrylamide gel, and subsequently transferred to polyvinylidene difluoride (PVDF) membrane. After the membranes were blocked with $5 \%$ non-fat milk solution, rabbit polyclonal anti-human LTB4DH and mouse polyclonal anti- $\beta$-actin antibodies were sequentially used to probe the cellular LTB4DH and $\beta$-actin. The bound antibodies were detected by horseradish peroxidase conjugated goat anti-rabbit IgG (Sigma). The activity of peroxidase on the blot was visualized by enhanced chemiluminescence (ECL) detection reagents (GE Healthcare, Sweden).

Colonogenic survival assay. Following the treatment with the active compounds isolated from two herbs, alone or in combination, for $24 \mathrm{~h}$, the cells were seeded at the density of 3000 cells/well in 6-well plate and cultured in modified growth medium MEM containing 5\% fetal bovine serum, herbal drugs, alone or in combination. Twelve days later, the cells were fixed by $70 \%$ ethanol at room temperature for $20 \mathrm{~min}$, air-dried for $30 \mathrm{~min}$, and then stained with crystal violet (Sigma-Aldrich) at $37^{\circ} \mathrm{C}$ for $60 \mathrm{~min}$. The excessive crystal violet was removed by washing with $1 \mathrm{X}$ PBS. After air-dried, the cell foci were enumerated on an inverted microscope. The colonogenic survival was calculated by the following equation: colonogenic survival $(\%)=$ (colony number/seeded cells) x 100\%. Three independent experiments were performed in parallel for each treatment. 

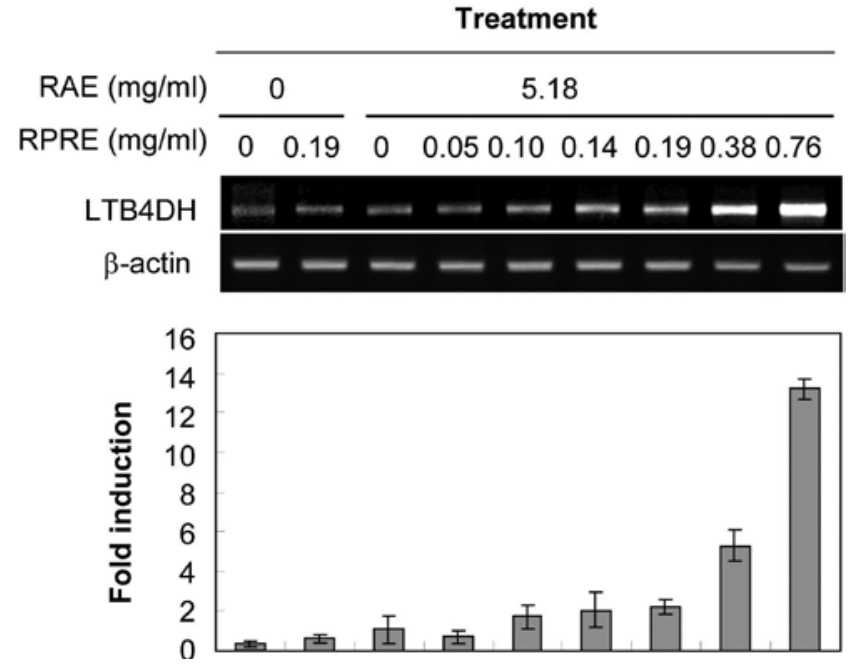

Figure 1. Induction LTB4DH expression is highly dependent on the combination of Radix Astragali extract and Radix Paeoniae Rubra extract. HepG2 cells were treated with the combination of Radix Astragali extract and Radix Paeoniae Rubra extract at different ratios of at $37^{\circ} \mathrm{C}$ for $24 \mathrm{~h}$. LTB4DH expression was detected by semi-quantitative RT-PCR using specific primers, whereas $\beta$-actin was detected as internal control. The signals were quantified and the mean values of three biological replicates were summarized. RAE, Radix Astragali extract; RPRE, Radix Paeoniae Rubra extract.

Anchorage-independent growth in soft agar. Prior to the assays of herbal drugs, the cell culture plates were filled with the bottom agarose ( $3 \mathrm{ml}$ per well). The bottom agarose was obtained by mixing equal volumes of sterilized $1.2 \%$ agarose and 2X MEM medium supplemented with $20 \%$ fetal bovine serum at $45^{\circ} \mathrm{C}$. The plates were placed at room temperature until the bottom agarose became solid. For the assays, the cells were treated with two herbal drugs, alone or in combination, as indicated, for $24 \mathrm{~h}$. Single cell suspension was made at the density of 3000 cells $/ \mathrm{ml}$ in the complete growth medium supplemented with two herbal drugs as indicated. The bottom agarose made previously was heated up to $45^{\circ} \mathrm{C}$ and the mixed with equal volume of the cell suspension. The mixture $(2 \mathrm{ml}$ per well) was immediately transferred onto the bottom agarose plate. The cells were cultured at $37^{\circ} \mathrm{C}$ in the incubator equilibrated with $5 \% \mathrm{CO}_{2}$ for 12 days. The colonies with a diameter $(>50 \mu \mathrm{m})$ were counted under an inverted microscope. The colonogenic fraction was calculated by the following equation: anchorage-independent growth rate $(\%)=$ (colony number/seeded cells) x $100 \%$. Three independent experiments were performed in parallel for each treatment.

Flow cytometric analysis of cell cycle. At the end of drug treatment, cells were detached by trypsin/EDTA and fixed by $70 \%$ ethanol. The intracellular RNAs were removed by RNase A in $1 \mathrm{X}$ PBS. Following the permeabilization with $0.1 \%$ Triton $\mathrm{X}-100$, the cells were stained with PI at a concentration of $50 \mu \mathrm{g} / \mathrm{ml}$. The cellular DNA contents were analyzed with a flow cytometer (BD Biosciences, CA, USA). The cell cycle phase distribution was quantified by ModFit LT 2.0 software (Verify Software House, http://www.vsh.com).

Statistical analysis. All data are presented as the means \pm SD for three independent experiments. Statistical analysis was performed by the paired Student's t-test. $\mathrm{p}<0.05$ was considered to be statistically significant.

\section{Results}

Induction of LTB4DH by the combination of Radix Astragali and Radix Paeoniae Rubra extracts. By investigating the pharmacological induction of LTB4DH, we recently found that LTB4DH could be induced by a combination of Radix Astragali and Radix Paeoniae Rubra extracts. In order to achieve the maximal induction of LTB4DH expression, we determined the LTB4DH expression in HepG2 cells treated with different combinations of these two herbal extracts. As shown in Fig. 1, neither Radix Paeoniae Rubra extract or Radix Astragali and Radix Paeoniae Rubra extract alone could induce LTB4DH expression up to a doses of $0.19 \mathrm{mg} / \mathrm{ml}$ and $5.18 \mathrm{mg} / \mathrm{ml}$, respectively. In the presence of Radix Astragali extract $(5.18 \mathrm{mg} / \mathrm{ml})$, however, LTB4DH induction was positively correlated with the increase in the concentration of Radix Paeoniae Rubra extract from 0 to $0.76 \mathrm{mg} / \mathrm{ml}$. Our results not only demonstrated for the first time the pharmacological induction of LTB4DH expression but also suggested that LTB4DH expression was induced via the coordination of different mechanisms.

Identification of the active compound from Radix Paeoniae Rubra extract. To characterize the active compounds contributing to the induction of LTB4DH expression, we described a bioactivity-guided fractionation procedure as illustrated in Fig. 2A. The dried aqueous herbal extracts were produced by Nong's Company for clinical application. We used water to re-dissolve the active compounds. Water-soluble materials were precipitated by ethanol to remove inorganic salts, polysaccharides and other polar compounds. The ethanol-soluble materials were subsequently separated by RP-HPLC on a C18 column into 13 fractions. Pertinent to our previous study, we assayed all fractions in combination with Radix Astragali extract for the activity to induce LTB4DH mRNA expression by semi-quantitative RT-PCR in HepG2 cell line as the cellular detector. As a result, LTB4DH mRNA was detected in Fraction No. 2 in Fig. 2B. Fraction No. 2 was further separated by RP-HPLC on a $\mathrm{C} 18$ column into 4 fractions. According to the bioassay shown in Fig. 2C, Fraction No. 3 in Fig. $2 \mathrm{C}$ showed the activity to induce LTB4DH expression. Fraction No. 3 showed the maximal UV absorption $\left(\lambda_{\max }\right)$ at 224 and $271 \mathrm{~nm}$. Further LC-MS analysis of Fraction No. 3 revealed

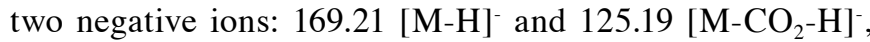
suggesting that this fraction may contain GA. In fact, ${ }^{1} \mathrm{H}$ NMR spectrum of this fraction was identical to that of GA (31). Based on the linear relationship of the peak area in HPLC profile with the concentration of commercial GA per injection, $\sim 16.4 \mathrm{~g}$ of GA was found to be present in $1 \mathrm{~kg}$ of dried Radix Paeoniae Rubra extract.

On the other hand, we found that LTB4DH induction required a relative larger amount of Radix Astragali extract than the amount of Radix Paeoniae Rubra extract. This finding suggested that either the concentration or the potency of the active compound RA-C in Radix Astragali extract could be quite low. Thus, we developed a bioactivity-guided fractionation strategy for rapid isolation of the active compound 
A

Dry Radix Paeoniae Rubra extract

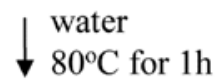

Aqueous extract

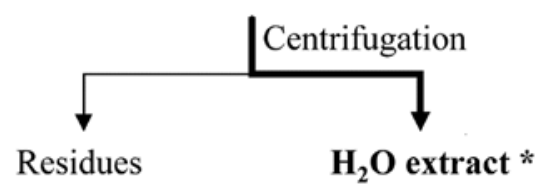

Precipitated by adding 3 volumes of $100 \%$ ethanol

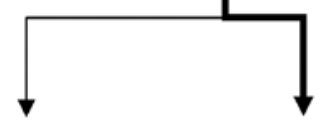

Residues Ethanol extract *

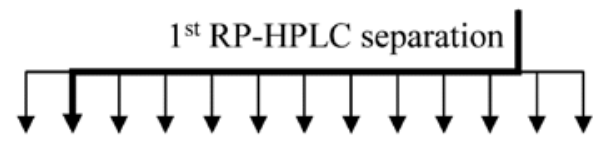

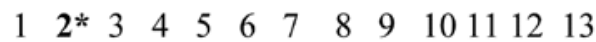

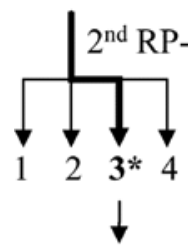

Gallic acid
B

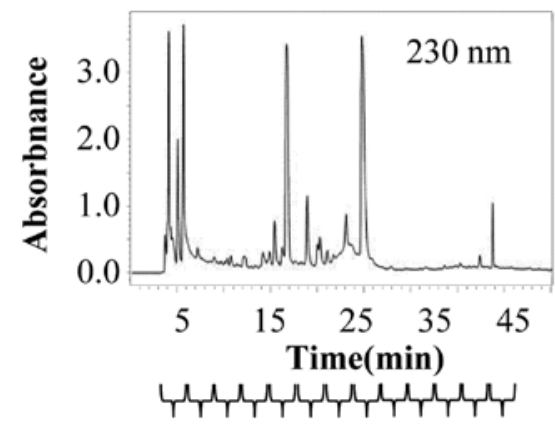

Fractions $\quad 012345678910111213$

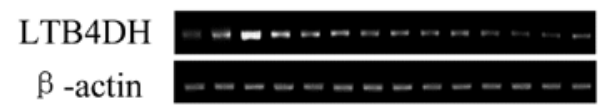

C

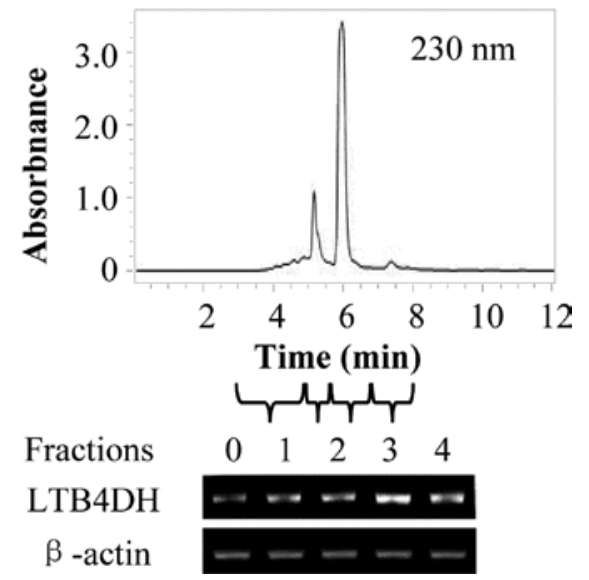

Figure 2. Bioactivity-guided isolation of the active compound from Radix Paeoniae Rubra extract. (A) Scheme illustrating the bioactivity-guided fractionation of the active compound from Radix Paeoniae Rubra extract. HPLC fractions were monitored for the absorbance at $230 \mathrm{~nm}$. Human hepatocellular carcinoma cell line HepG2 was used as the cellular detector to assay all fractions generated in this process for LTB4DH induction. *, Fractions or compounds showed the activity in LTB4DH induction. (B) The first round of HPLC fractionation and RT-PCR detection of LTB4DH induction. After the aqueous Radix Paeoniae Rubra extract was precipitated by $70 \%$ ethanol, the soluble materials were separated by RP-HPLC on a C18 column as described in Materials and methods. Thirteen fractions were collected and determined for LTB4DH induction in HepG2 cells by semi-quantitative RT-PCR. Representative HPLC profile and RT-PCR analysis were shown. (C) The second round of HPLC fractionation and RT-PCR detection of LTB4DH induction. The active fraction No. 2 extract was separated by RP-HPLC on a C18 column as described in Materials and methods. Four fractions were collected and subsequently determined for LTB4DH induction in HepG2 cells by semi-quantitative RT-PCR. Representative HPLC profile and RT-PCR analysis are shown.

RA-C from Radix Astragali extract at a larger scale. This fractionation was initiated with $2 \mathrm{~kg}$ of dried Radix Astragali extract by ethanol extraction. The resulted ethanolic extract was separated by RP-HPLC on a Waters XBridge Prep Shield RP18 column (5 $\mu \mathrm{m}$ OBD 19x250 mm). The active fraction was further separated by RP-HPLC on an Alitima C18 column (250x4.6 mm I.D., $5 \mu \mathrm{m})$ as described previously (32). As a result, the active compound RA-C was purified from Radix Astragali extract. The identification of the active compound RA-C from Radix Astragali will be published elsewhere.

Verification of LTB4DH induction by commercial GA in combination with purified $R A-C$. Following the identification of GA as the active compound from Radix Paeoniae Rubra extract, we employed the combination of commercial GA (Sigma-Aldrich) with purified RA-C in the induction of LTB4DH expression. LTB4DH mRNA expression was verified by semi-quantitative RT-PCR whereas LTB4DH protein was detected by Western blotting using the specific antibody. As shown in Fig. 3A and B, LTB4DH expression was significantly induced by the combination of commercial GA and purified RA-C, whereas either GA or purified RA-C alone did not show strong activity. Moreover, LTB4DH expression was induced by the combination of commercial GA and purified RA-C in a time-dependent manner. LTB4DH mRNA was detectable as early as $3 \mathrm{~h}$ after the treatment was initiated, suggesting that LTB4DH induction is an early response to the treatment with the combination of commercial GA and purified RA-C.

Inhibition of in vitro oncogenic transformation of HepG2 cells by $G A$ and purified $R A-C$. It is well-known that transformed cells have better ability to survive and grow even in an anchorage-independent manner (33). To examine the effects of GA and purified RA-C combination on in vitro oncogenic 
$\mathbf{A}$
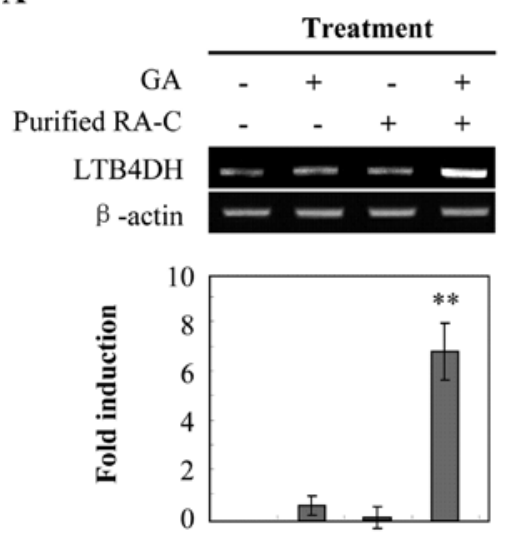

B
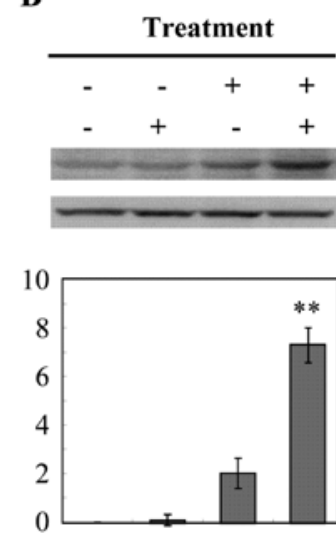

C
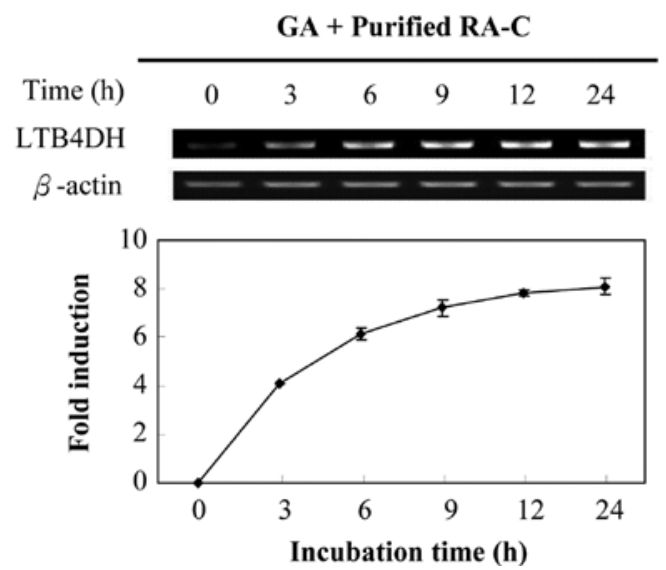

Figure 3. Verification of LTB4DH induction by commercial GA and purified RA-C. (A) RT-PCR detection of LTB4DH induction. The cells were treated with GA $(7 \mu \mathrm{g} / \mathrm{ml})$ and purified RA-C $(28 \mu \mathrm{g} / \mathrm{ml})$, alone or in combination, for $24 \mathrm{~h}$. Total cellular RNAs were extracted. LTB4DH expression was detected by RT-PCR using specific primers as described Materials and methods. (B) Western blot analysis of LTB4DH induction. The cells were treated with GA $(7 \mu \mathrm{g} / \mathrm{ml})$ and purified RA-C $(28 \mu \mathrm{g} / \mathrm{ml})$ similarly as described in (A). The cellular proteins were extracted and resolved by $10 \%$ SDS-PAGE. LTB4DH expression was detected by Western blotting using specific antibodies (Abnova) as described Materials and methods. (C) Time course of LTB4DH induction by the combination of GA and purified RA-C. The cells were treated with the combination of GA $(7 \mu \mathrm{g} / \mathrm{ml})$ and purified RA-C $(28 \mu \mathrm{g} / \mathrm{ml})$ for indicated time points $(0,3,6,9,12,24 \mathrm{~h})$. Total cellular RNAs were extracted. LTB4DH expression was detected by RT-PCR using specific primers. The mean values of three independent experiments were shown. Significance analysis was performed by two-tail paired Student's t-test. ${ }^{* *}$ p $<0.01$ compared with control.

transformation, HepG2 cells were treated with GA and purified RA-C, alone or in combination, for $24 \mathrm{~h}$. The cells were examined for the proliferation, focus formation and anchorage-independent growth in soft agar. As shown in Fig. 4, the combination of GA and purified RA-C effectively inhibited the growth, focus formation and anchorageindependent growth of HepG2 cells. We found that the GI50 value of GA in HepG2 cells was $26 \mu \mathrm{g} / \mathrm{ml}$, whereas purified RA-C is almost non-cytotoxic in HepG2 cells even at a dose of $800 \mu \mathrm{g} / \mathrm{ml}$. It was not surprising that the combination of GA and purified RA-C almost abolish the growth of HepG2 cells whereas purified RA-C did not show any inhibition (Fig. 4A). The inhibitory effect of GA is consistent with the results of a recent study, which demonstrated the cytotoxicity
A

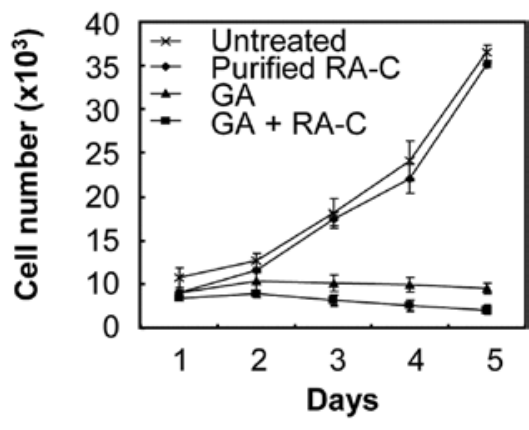

B
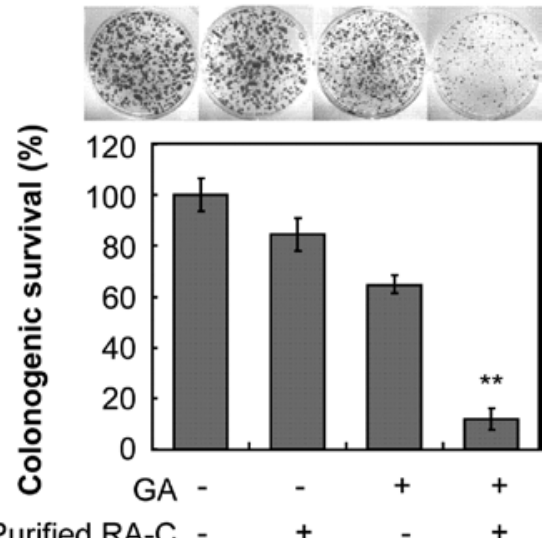

Purified RA-C - + $+\quad+$

C

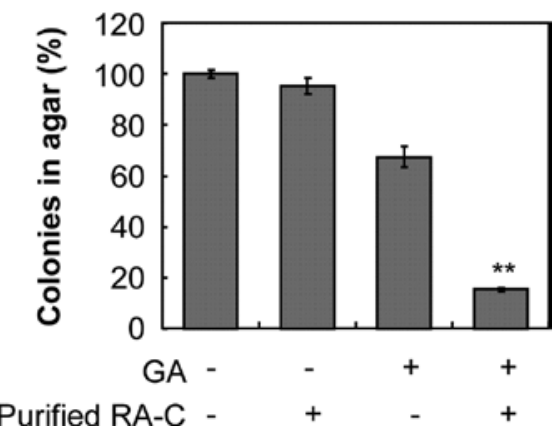

Figure 4. Inhibitory effect of LTB4DH inducers GA and purified RA-C on the transforming activity and proliferation of HepG2 cells. (A) Effect of GA and purified RA-C on the growth of HepG2 cells. HepG2 cells were treated with GA $(7 \mu \mathrm{g} / \mathrm{ml})$ and purified RA-C $(28 \mu \mathrm{g} / \mathrm{ml})$, alone or in combination, for indicated time points over a period of 5 days. The cell number was counted by haemocytometer. (B) Colonogenic survival assay. Following the exposure to GA $(7 \mu \mathrm{g} / \mathrm{ml})$ and purified RA-C $(28 \mu \mathrm{g} / \mathrm{ml})$, alone or in combination, for $24 \mathrm{~h}, \mathrm{HepG} 2$ cells (3000 cells/well) were seeded in 6-well plate in triplicates. The cells were cultured in the presence of 5\% fetal bovine serum for 12 days. The cell foci were stained with $0.5 \%$ crystal violet in $20 \%$ ice-cold methanol as described in Materials and methods. The stained cell foci were counted under light microscopy. (C) Anchorageindependent growth in soft agar. Following the exposure to GA $(7 \mu \mathrm{g} / \mathrm{ml})$ and purified RA-C ( $28 \mu \mathrm{g} / \mathrm{ml})$, alone or in combination, for $24 \mathrm{~h}$, HepG2 cells (3000 cells/well) in top agarose were seeded onto bottom agarose supplemented with complete growth medium in a 6-well plate (three wells per treatment) for 12 days. The cell colonies were counted under an inverted phase microscope. Significance analysis was performed by the paired Student's t-test. ${ }^{* *} \mathrm{p}<0.01$ vs. control.

of GA in testicular cell lines (34). Of note, the combination of GA and purified RA-C inhibited the focus formation and anchorage-independent growth of HepG2 cells by $76-83 \%$ and $82-88 \%$, respectively, whereas either GA or purified RA-C alone did not show much activity. These results suggest that GA 
A

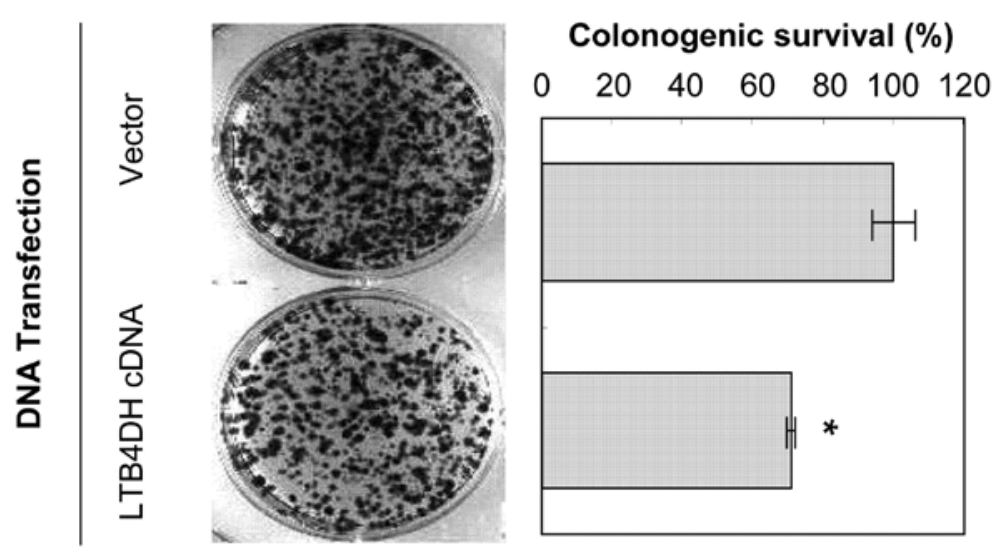

B

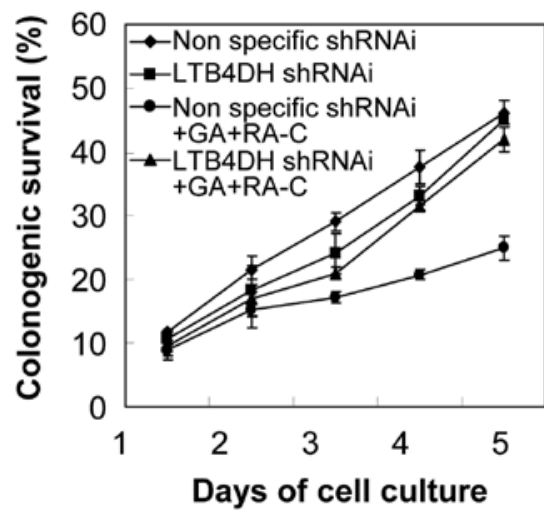

C

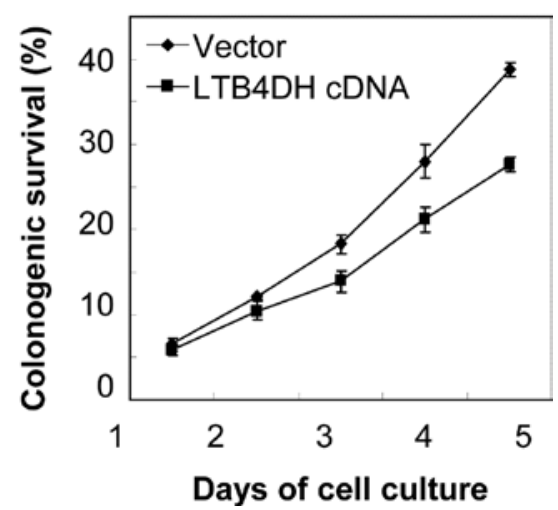

Figure 5. Inhibitory role of LTBDH in the cell growth of HepG2 cells. (A) Effect of ectopic expression of LTBDH on the colonogenic survival of HepG2 cells. HepG2 cell line stably overexpressing LTB4DH was established as described in Materials and methods, whereas the cell line stably transfected with vector alone was used as a control. The cells (3000 cells/well) were seeded in 6-well plate in triplicate and cultured in the presence of 5\% fetal bovine serum for 12 days. The cell foci were stained with $0.5 \%$ crystal violet in $20 \%$ ice-cold methanol and then counted by light microscopy. The results were expressed as the the mean \pm SD ( $=3$ ). * $p<0.05$ vs. transfection with vector alone. (B) Effect of LTB4DH transfection on the growth of HepG2 cells. The cell line stably overexpressing LTB4DH was established as described in Materials and methods, whereas the cell line stably transfected with vector alone was used as a control. Cells were seeded at 3x10\%/well in 6-well plate and the cell numbers were counted by a hemocytometer everyday over a period of 5 days. (C) Antagonizing effect of LTB4DH specific RNAi on the suppressive activity of GA and RA-C combination in cancer cells. HepG2 cell lines stably carrying LTB4DH-specific shRNA (pRS-LTB4DH) and nonspecific shRNA (pRS-Ctrl) were established as described in Materials and methods. Cells were treated with GA $(7 \mu \mathrm{g} / \mathrm{ml})$ and purified RA-C $(28 \mu \mathrm{g} / \mathrm{ml})$ in combination for $24 \mathrm{~h}$ and then seeded at $3 \times 10^{4}$ cells/well in a 6 -well plate in triplicates. The cell growth was monitored by a hemocytometer everyday over a period of 5 days.

and purified RA-C may inhibit the in vitro oncogenic transformation of HepG2 cells in a synergistic manner.

Evidence for the role of $L T B 4 D H$ in the anticancer activity of the combination of $G A$ and purified $R A-C$. It was recently demonstrated that ectopic overexpression of LTB4DH suppressed the proliferation of breast cancer cells (17). By introducing LTB4DH cDNA into HepG2 cells, we verified the inhibitory effect of LTB4DH on the colonogenic survival and growth of hepatoma cells (Fig. 5A and B). Through careful comparison of Fig. 5A and B and Fig. 4A-C, it appeared that ectopic overexpression of LTB4DH mimicked the effect of GA and purified RA-C on the in vitro oncogenic transformation and cell growth of HepG2. We further addressed the question of whether the induction of LTB4DH expression is the only mechanism underlying the anticancer activity of GA and purified RA-C combination. Two stable cell lines carrying either LTB4DH specific shRNAi construct or non-specific control were established and exposed to the combination of GA and purified RA-C over a period of five consecutive days. The growth of the cells under treatment was monitored on a daily basis. As shown in Fig. 5C, the drugs inhibited the growth of non-specific shRNAi transfected cells and did not significantly alter the growth of LTB4DH specific shRNAi transfected cells. Our pilot experiments showed that the combination of GA and purified RA-C failed to induce LTB4DH expression in the cells stably transfected with LTB4DH-specific shRNAi (data not shown). Moreover, when the induction of LTB4DH expression is blocked by specific shRNAi, the combination of GA and purified RA-C could not inhibit the growth of HepG2 cells transfected with LTB4DHspecific shRNAi. In contrast, non-specific shRNAi did not antagonize the induction of LTB4DH expression by the combination of GA and purified RA-C. Thus, these results 

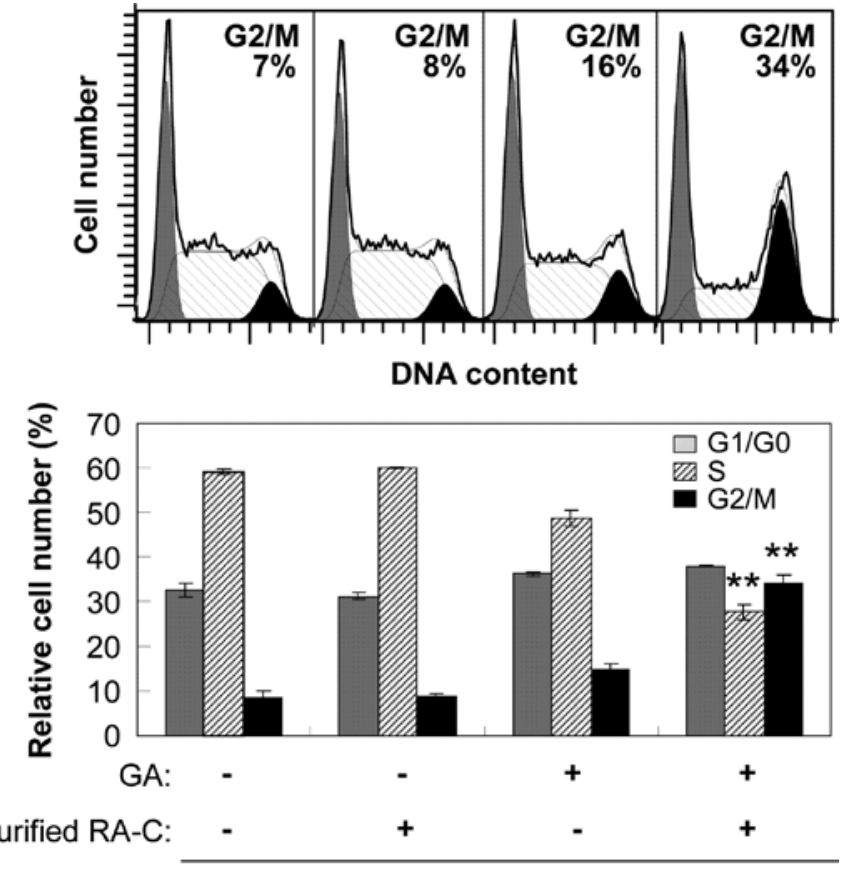

Treatment

Figure 6. Induction of cell cycle arrest by GA and purified RA-C in combination. HepG2 cells were treated by GA $(7 \mu \mathrm{g} / \mathrm{ml})$ and purified RA-C $(28 \mu \mathrm{g} / \mathrm{ml})$, alone or in combination, for $48 \mathrm{~h}$. Treated cells were fixed by ice cold ethanol $(70 \%)$ overnight. Fixed cells were stained with propidium iodide and analyzed by flow cytometry on a FACS Calibur cytometer. The cell population in different cell cycle phases was calculated by ModFit LT The results represented the mean \pm SD of three biological replicates. Representative histograms of cell cycle are shown. Test of significance was performed by the paired Student's t-test, ${ }^{* *} \mathrm{p}<0.01$ vs. control.

demonstrated a critical role of LTB4DH in the anticancer activity of GA and purified RA-C combination.

Synergistic induction of LTB4DH expression results in cell cycle arrest. To explore the mechanism underlying the action of GA and purified RA-C combination in the cells, we performed a cell cycle analysis of HepG2 cells treated or untreated with these two compounds. According to the cell cycle profiling, it appeared that the cells were arrested at G2/M phase by the combination of GA and purified RA-C, whereas either GA or purified RA-C alone did not alter the cell cycle in HepG2 cells (Fig. 6). Our results suggested that synergistic induction of cell cycle arrest by the combination of GA and purified RA-C might be a novel mechanism leading to the inhibition of the cell proliferation of HepG2 cells.

\section{Discussion}

Oncogenic transformation of human cells is triggered by genetic and epigenetic alterations of various genes and proteins involved in DNA-metabolizing, cell signaling, cell proliferation and cell death $(35,36)$. Earlier anticancer strategy focused on the development of cytotoxic drugs, and however recent effort has been directed to the development of mechanism-specific anticancer drugs $(19,37,38)$. One of the effective anticancer mechanisms is to activate tumor suppressor genes in cancer patients $(19,39)$. LTB4DH was
(A) LTB4 Signaling

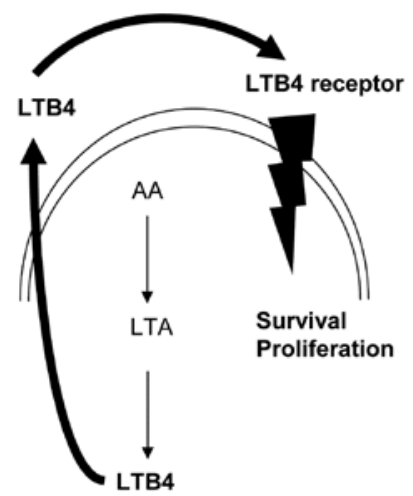

(B) Effect of LTB4DH on LTB4 signaling

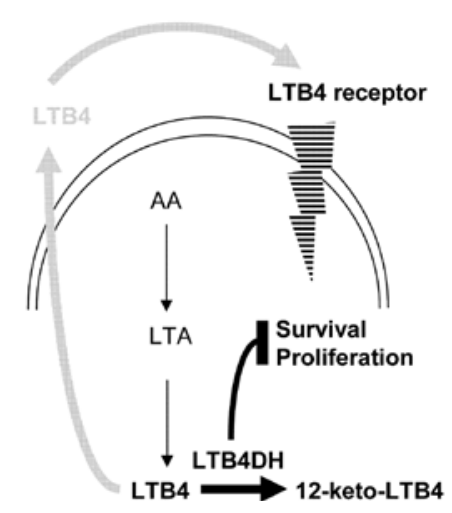

Figure 7. Metabolism and signaling of LTB4 in human cells. (A) Signaling of LTB4 in human cells. The cell membrane arachidonic acids are converted to LTA by 5 -LOX. The intermediate LTA is hydrolyzed to LTB4 by LTA hydrolase. LTB4 stimulates the survival and proliferation of cells via activating LTB4 receptor. (B) Inhibition of LTB4 signaling by LTB4DH. LTB4DH catalyzes the oxidation of proinflammatory mediator LTB4 to less active 12-keto-LTB4, thereby reducing the secretion of LTB4 to activate the cell surface LTB4 receptor. As a result, LTB4-mediated survival and proliferating signals are suppressed.

isolated as dithiolethione-inducible gene-1 (DIG-1) and thereby classified as a chemopreventive and tumor suppressive gene (18). In addition, dithiolethiones could up-regulate various phase 2 detoxifying enzymes and inhibit chemically induced carcinogenesis (40). Of note, a large number of botanical compounds isolated from medicinal herbs also induce phase 2 detoxifying enzymes (41-43). Thus, we recently searched for novel LTB4DH-inducers from medicinal plants historically used as anticancer therapy in traditional Chinese medicine. In the present study, we described a bioactivityguided fractionation strategy for rapid isolation of GA and RA-C as LTB4DH-inducers from the herbs Radix Paeoniae Rubra and Radix Astragali. The central findings of the present study are that GA and purified RA-C synergistically induced LTB4DH expression and rendered the inhibition of in vitro oncogenic transformation of human HepG2 cells, as a surrogate of human hepatocellular carcinoma.

Gene transcription is regulated by the coordination of various transcription factors, enhancers and suppressors $(44,45)$. It is a daunt task to perform rapid and simultaneous characterization of the genes regulated by multiple molecules in one-pot. Presumably, different compounds trigger a complex cross-talk between specific transcriptional regulatory elements. We recently described a genome-wide biological response fingerprinting (BioReF) approach for simultaneous identification of the genes regulated by complex herbal formulations in human cells $(28,46)$. By assaying the change of specific genes, we further developed a step-wise deletion strategy for the identification of the active ingredients in the formulation as described (30). As a result, we successfully characterized three active ingredients for the induction of heme oxygenase-1 expression. The present study represents our continuing effort to investigate the pharmacological induction of LTB4DH. Through a similar procedure, we identified Radix Astragali and Radix Paeoniae Rubra as the potential ingredients for the induction of LTB4DH. By 
assaying the induction of LTB4DH mRNA using RT-PCR technique, GA and RA-C were isolated and identified from the herbs Radix Paeoniae Rubra and Radix Astragali, respectively. Following the verification of commercial GA (Fig. 3), we also found that propyl gallate in combination with purified RA-C could equally induce LTB4DH expression, whereas oltipraz and caffeic acid did not show any activity (data not shown). Our results did not exclude the possibility of that oltipraz metabolites may be able to induce LTB4DH expression as reported previously (18).

GA is a potent antioxidant and anti-inflammatory reagent widely present, in free form or as conjugates, in plants, fruits and vegetables (47). GA is also found to induce apotosis by modulating two key intracellular signaling pathways, reactive oxygen species (ROS)- and calcium-mediated signaling pathways, respectively (48). It is important to note that GA selectively induced apoptosis in a variety of cancer cell lines but not in non-cancerous cells (49,50). Different alkyl, gallamide and p-tyrosol derivatives of GA also inhibit the proliferation and induce cell cycle arrest in cancer cells such as human leukemia HL-60 cells and HeLa cells (51-53). The anticancer activity of GA is mediated by multiple proapoptotic mechanisms: (1) by activating Fas- and mitochondrialmediated pathways $(54,55)$; (2) by up-regulating pro-apoptotic proteins, Bax, activating caspases and down-regulating antiapoptotic proteins such as Bcl-2 and Xiap; (3) by suppressing the survival-promoting signaling pathways such as Akt/mTOR pathway, delaying the expression of pro-apoptosis related proteins in non-cancerous cells and enhancing antiapoptotic potential in normal human lymphocytes via a Bcl-2 independent mechanism $(50,56)$; and (4) by inducing cell cycle arrest and apoptosis in human prostate carcinoma DU145 cells (57). Although GA showed slight cytotoxicity in human blood lymphocytes, GA did not induce toxicity in rats at the dose of $119-128 \mathrm{mg} / \mathrm{ml}$ (58). These results further consolidated that GA possesses strong cancer-selective cytotoxicity. Normal cells such as hepatocytes and keratinocytes likely secreted a protein-like molecule to antagonize GA-induced apoptosis (53). On the other hand, Radix Astragali extract is generally considered to be nontoxic to patients even at a high dose of $120 \mathrm{~g} /$ day $(28,59)$. The anticancer activity of Radix Astragali is principally mediated by modulating the immunological responses inside the cells. Our results also confirmed that GA inhibited the growth of human liver cancer HepG2 and such inhibition was only slightly enhanced by purified RA-C (Fig. 4A).

LTB4DH was recently suggested as a chemopreventive and tumor suppressive gene. Based on the assays of the focus formation and anchorage-independent growth, we demonstrated that the combination of GA and purified RA-C strongly inhibited the in vitro oncogenic transformation of HepG2 cells (Fig. 4B and C). Through ectopic expression and shRNAi-mediated knockdown of LTB4DH mRNA, LTB4DH was proven to play a critical role in the anticancer activity of GA and purified RA-C in combination (Fig. 5). It is wellknown that tumor suppressor genes often induced cell cycle arrest in cancer cells (60). Our results also showed that LTB4DH induction resulted in the inhibition of cell proliferation and cell cycle arrest in HepG2 cells (Fig. 6). In particular, the stimulation with GA and purified RA-C induced LTB4DH expression, conferring the concomitant increase of cell population at $\mathrm{G} 2 / \mathrm{M}$ phase and decrease of cell population at $\mathrm{S}$ phase. These results further confirmed the tumor suppressor characteristics of LTB4DH. Finally, the effect of LTB4DH on LTB4-mediated cell signals is summarized in Fig. 7A. Briefly, LTB4 activates its cell surface receptor to promote cell survival and stimulate proliferation of cancer cells. Upon the stimulation by the combination of GA and purified RA-C, LTB4DH is induced to catalyze the metabolism of LTB4 to less active 12-oxo-LTB4 (Fig. 7B). Consequently, LTB4mediated signals are no longer generated and the cancer cells undergo apoptosis.

In summary, the present study described a bioactivityguided fractionation procedure for rapid isolation of GA and RA-C from Radix Paeoniae Rubra and Radix Astragali extracts as novel LTB4DH inducers. Commercial GA and purified RA-C were found to synergistically induce LTB4DH expression in a time- and concentration-dependent manner. A key finding was that the combination of GA and purified $\mathrm{RA}-\mathrm{C}$ attenuated the in vitro oncogenic transformation of HepG2 cells and induced cell cycle arrest. By ectopic expression and shRNAi-mediated knockdown of LTB4DH, we provided evidence to support that induction of LTB4DH is a key mechanism underlying the anticancer activity of the combination of GA and RA-C. Our results suggest that pharmacological induction of LTB4DH by the combination of GA and RA-C may represent a novel anticancer mechanism.

\section{Acknowledgements}

This work was partly supported by a General Research Fund (GRF) grant (HKU774307 M) from the Research Grants Council of Hong Kong (to J.R.) and two internal Seed Funds for Basic Research Programme from University of Hong Kong.

\section{References}

1. Samuelsson B and Claesson HE: Leukotriene B4: biosynthesis and role in lymphocytes. Adv Prostaglandin Thromboxane Leukot Res 20: 1-13, 1990.

2. Yokomizo T, Izumi T and Shimizu T: Leukotriene B4: metabolism and signal transduction. Arch Biochem Biophys 385: 231-241, 2001.

3. Bittner S and Wielckens K: Glucocorticoid-induced lymphoma cell growth inhibition: the role of leukotriene B4. Endocrinology 123: 991-1000, 1988

4. Yokomizo T, Kato K, Terawaki K, Izumi T and Shimizu T: A second leukotriene $\mathrm{B}(4)$ receptor, BLT2. A new therapeutic target in inflammation and immunological disorders. J Exp Med 192: 421-432, 2000.

5. Avis IM, Jett M, Boyle T, et al: Growth control of lung cancer by interruption of 5-lipoxygenase-mediated growth factor signaling. J Clin Invest 97: 806-813, 1996.

6. Chen X, Li N, Wang S, et al: Leukotriene A4 hydrolase in rat and human esophageal adenocarcinomas and inhibitory effects of bestatin. J Natl Cancer Inst 95: 1053-1061, 2003.

7. Kato K, Yokomizo T, Izumi T and Shimizu T: Cell-specific transcriptional regulation of human leukotriene $\mathrm{B}(4)$ receptor gene. J Exp Med 192: 413-420, 2000.

8. Earashi $M$, Noguchi $M$ and Tanaka $M$ : In vitro effects of eicosanoid synthesis inhibitors in the presence of linoleic acid on MDA-MB-231 human breast cancer cells. Breast Cancer Res Treat 37: 29-37, 1996.

9. Ihara A, Wada K, Yoneda M, Fujisawa N, Takahashi H and Nakajima A: Blockade of leukotriene B4 signaling pathway induces apoptosis and suppresses cell proliferation in colon cancer. J Pharmacol Sci 103: 24-32, 2007. 
10. Tai HH, Ensor CM, Tong M, Zhou H and Yan F: Prostaglandin catabolizing enzymes. Prostaglandins Other Lipid Mediat 68-69: 483-493, 2002.

11. Dick RA, Kwak MK, Sutter TR and Kensler TW: Antioxidative function and substrate specificity of NAD(P)H-dependent alkenal/one oxidoreductase. A new role for leukotriene B4 12-hydroxydehydrogenase/15-oxoprostaglandin 13-reductase. J Biol Chem 276: 40803-40810, 2001.

12. Back M, Bu DX, Branstrom R, Sheikine Y, Yan ZQ and Hansson GK: Leukotriene B4 signaling through NF-kappaB-dependent BLT1 receptors on vascular smooth muscle cells in atherosclerosis and intimal hyperplasia. Proc Natl Acad Sci USA 102: 17501-17506, 2005.

13. Radmark O, Shimizu T, Jornvall H and Samuelsson B: Leukotriene A4 hydrolase in human leukocytes. Purification and properties. J Biol Chem 259: 12339-12345, 1984.

14. Tong M, Ding Y and Tai HH: Reciprocal regulation of cyclooxygenase-2 and 15-hydroxyprostaglandin dehydrogenase expression in A549 human lung adenocarcinoma cells. Carcinogenesis 27: 2170-2179, 2006.

15. Yan M, Rerko RM, Platzer P, et al: 15 -Hydroxyprostaglandin dehydrogenase, a COX-2 oncogene antagonist, is a TGF-betainduced suppressor of human gastrointestinal cancers. Proc Natl Acad Sci USA 101: 17468-17473, 2004.

16. Wolf I, O'Kelly J, Rubinek T, et al: 15-hydroxyprostaglandin dehydrogenase is a tumor suppressor of human breast cancer. Cancer Res 66: 7818-7823, 2006.

17. Zhao Y, Weng CC, Tong M, Wei J and Tai HH: Restoration of leukotriene B(4)-12-hydroxydehydrogenase/15-oxo-prostaglandin 13-reductase (LTBDH/PGR) expression inhibits lung cancer growth in vitro and in vivo. Lung Cancer 68: 161-169, 2010.

18. Primiano T, Li Y, Kensler TW, Trush MA and Sutter TR: Identi cation of dithiolethione-inducible gene-1 as a leukotriene B4 12-hydroxydehydrogenase: implications for chemoprevention. Carcinogenesis 19: 999-1005, 2008

19. Efferth T, Kahl S, Paulus K, et al: Phytochemistry and pharmacogenomics of natural products derived from traditional Chinese medicine and Chinese materia medica with activity against tumor cells. Mol Cancer Ther 7: 152-161, 2008.

20. Galati G and O'Brien PJ: Potential toxicity of flavonoids and other dietary phenolics: significance for their chemopreventive and anticancer properties. Free Radic Biol Med 37: 287-303, 2004.

21. Surh YJ: Cancer chemoprevention with dietary phytochemicals. Nat Rev Cancer 3: 768-780, 2003.

22. Zhan LY, Xia ZY, Chen C and Wang XY: Effect of Radix Paeoniae Rubra on the expression of HO-1 and iNOS in rats with endotoxin-induced acute lung injury. Chin J Traumatol 9: 181-186, 2006.

23. Liu EH, Qi LW, Li B, et al: High-speed separation and characterization of major constituents in Radix Paeoniae Rubra by fast high-performance liquid chromatography coupled with diode-array detection and time-of-flight mass spectrometry. Rapid Commun Mass Spectrom 23: 119-130, 2009.

24. Cui R, He J, Wang B, et al: Suppressive effect of Astragalus membranaceus Bunge on chemical hepatocarcinogenesis in rats. Cancer Chemother Pharmacol 51: 75-80, 2003.

25. Sinclair S: Chinese herbs: a clinical review of Astragalus, Ligusticum, and Schizandrae. Altern Med Rev 3: 338-344, 1998

26. Wong BY, Lau BH, Tadi PP and Teel RW: Chinese medicinal herbs modulate mutagenesis, DNA binding and metabolism of aflatoxin B1. Mutat Res 279: 209-216, 1992.

27. Rios J and Waterman P: A review of the pharmacology and toxicology of Astragalus. Phytother Res 11: 411-418, 1997.

28. Rong J, Tilton R, Shen J, et al: Genome-wide biological response fingerprinting (BioReF) of the Chinese botanical formulation ISF-1 enables the selection of multiple marker genes as a potential metric for quality control. J Ethnopharmacol 113: 35-44, 2007.

29. Qi H, Siu SO, Chen Y, et al: Senkyunolides reduce hydrogen peroxide-induced oxidative damage in human liver HepG2 cells via induction of heme oxygenase-1. Chem Biol Interact 183: 380-389, 2010.

30. Rong JH, Cheung CYH, Lau ASY, Shen JG, Tam PKH and Cheng YC: Induction of heme oxygenase-1 by traditional Chinese medicine formulation ISF-1 and its ingredients as a cytoprotective mechanism against oxidative stress. Int J Mol Med 21: 405-411, 2008.

31. Lu J, Wei Y and Yuan Q: Preparative separation of gallic acid from Chinese traditional medicine by high-speed countercurrent chromatography and followed by preparative liquid chromatography. Separation Purification Technol 55: 40-43, 2007.
32. Xu S, Yang L, Tian R, et al: Species differentiation and quality assessment of Radix Paeoniae Rubra (Chi-shao) by means of high-performance liquid chromatographic fingerprint. J Chromatogr A 1216: 2163-2168, 2009.

33. Wang LH: Molecular signaling regulating anchorage-independent growth of cancer cells. Mt Sinai J Med 71: 361-367, 2004.

34. Park W, Chang MS, Kim H, et al: Cytotoxic effect of gallic acid on testicular cell lines with increasing $\mathrm{H}_{2} \mathrm{O}_{2}$ level in GC-1 spg cells. Toxicol In Vitro 22: 159-163, 2008.

35. Ralph SJ, Rodriguez-Enriquez S, Neuzil J, Saavedra E and Moreno-Sanchez R: The causes of cancer revisited: 'mitochondrial malignancy' and ROS-induced oncogenic trans-formation - why mitochondria are targets for cancer therapy. Mol Aspects Med 31: 145-170, 2010.

36. Schinzel AC and Hahn WC: Oncogenic transformation and experimental models of human cancer. Front Biosci 13: 71-84, 2008.

37. Colotta F: Anticancer drug discovery and development. Adv Exp Med Biol 610: 19-42, 2008.

38. Powis G: Signalling pathways as targets for anticancer drug development. Pharmacol Ther 62: 57-95, 1994.

39. Martinez JD: Restoring p53 tumor suppressor activity as an anticancer therapeutic strategy. Future Oncol 6: 1857-1862, 2010.

40. Kensler TW, Curphey TJ, Maxiutenko Y and Roebuck BD: Chemoprotection by organosulfur inducers of phase 2 enzymes: dithiolethiones and dithiins. Drug Metabol Drug Interact 17: $3-22,2000$

41. Boik J: Natural Compounds in Cancer Therapy. Oregon Medical Press, 2001.

42. Efferth T, Li PC, Konkimalla VS and Kaina B: From traditional Chinese medicine to rational cancer therapy. Trends Mol Med 13: 353-361, 2007.

43. Newman DJ and Cragg GM: Natural products as sources of new drugs over the last 25 years. J Nat Prod 70: 461-477, 2007.

44. Granneman S and Baserga SJ: Crosstalk in gene expression: coupling and co-regulation of rDNA transcription, pre-ribosome assembly and pre-rRNA processing. Curr Opin Cell Biol 17: 281-286, 2005.

45. Doppler W, Geymayer S and Weirich HG: Synergistic and antagonistic interactions of transcription factors in the regulation of milk protein gene expression. Mechanisms of cross-talk between signalling pathways. Adv Exp Med Biol 480: 139-146, 2000.

46. Lee DC, Yang CL, Chik SC, et al: Bioactivity-guided identification and cell signaling technology to delineate the immunomodulatory effects of Panax ginseng on human promonocytic U937 cells. J Transl Med 7: 34, 2009.

47. Kroes BH, van den Berg AJ, Quarles van Ufford HC, van Dijk H and Labadie RP: Anti-inflammatory activity of gallic acid. Planta Med 58: 499-504, 1992.

48. Inoue M, Suzuki R, Koide T, Sakaguchi N, Ogihara Y and Yabu Y: Antioxidant, gallic acid, induces apoptosis in HL-60RG cells. Biochem Biophys Res Commun 204: 898-904, 1994.

49. Sarkaria JN, Busby EC, Tibbetts RS, et al: Inhibition of ATM and ATR kinase activities by the radiosensitizing agent, caffeine. Cancer Res 59: 4375-4382, 1999.

50. Faried A, Kurnia D, Faried LS, et al: Anticancer effects of gallic acid isolated from Indonesian herbal medicine, Phaleria macrocarpa (Scheff.) Boerl, on human cancer cell lines. Int J Oncol 30: 605-613, 2007.

51. Dodo K, Minato T, Noguchi-Yachide T, Suganuma $M$ and Hashimoto Y: Antiproliferative and apoptosis-inducing activities of alkyl gallate and gallamide derivatives related to (-)-epigallocatechin gallate. Bioorg Med Chem 16: 7975-7982, 2008.

52. Ahn EY, Jiang Y, Zhang Y, et al: Cytotoxicity of p-tyrosol and its derivatives may correlate with the inhibition of DNA replication initiation. Oncol Rep 19: 527-534, 2008.

53. Isuzugawa K, Ogihara Y and Inoue M: Different generation of inhibitors against gallic acid-induced apoptosis produces different sensitivity to gallic acid. Biol Pharm Bull 24: 249-253, 2001.

54. Kuo PL, Hsu YL, Sung SC, Ni WC, Lin TC and Lin CC: Induction of apoptosis in human breast adenocarcinoma MCF-7 cells by pterocarnin A from the bark of Pterocarya stenoptera via the Fas-mediated pathway. Anticancer Drugs 18: 555-562, 2007.

55. Miyamoto Y, Haylor JL and El Nahas AM: Cellular toxicity of catechin analogues containing gallate in opossum kidney proximal tubular (OK) cells. J Toxicol Sci 29: 47-52, 2004. 
56. Sohi K, Mittal N, Hundal M and Khanduja K: Gallic acid an antioxidant, exhibits anti-apoptotic potential in normal human lymphocytes: a Bcl-2 independent mechanism. J Nutr Sci Vitaminol 49: 221-227, 2003.

57. Agarwal C, Tyagi A and Agarwal R: Gallic acid causes inactivating phosphorylation of $\mathrm{cdc} 25 \mathrm{~A} / \mathrm{cdc} 25 \mathrm{C}-\mathrm{cdc} 2$ via ATM-Chk 2 activation, leading to cell cycle arrest, and induces apoptosis in human prostate carcinoma DU145 cells. Mol Cancer Ther 5: 3294-3302, 2006.

58. Niho N, Shibutani M, Tamura T, et al: Subchronic toxicity study of gallic acid by oral administration in F344 rats. Food Chem Toxicol 39: 1063-1070, 2001.
59. Cai G, Liu B, Liu W, et al: Buyang Huanwu Decoction can improve recovery of neurological function, reduce infarction volume, stimulate neural proliferation and modulate VEGF and Flk1 expressions in transient focal cerebral ischaemic rat brains. J Ethnopharmacol 113: 292-299, 2007.

60. Zhang X, Xu LS, Wang ZQ, et al: ING4 induces G2/M cell cycle arrest and enhances the chemosensitivity to DNA-damage agents in HepG2 cells. FEBS Lett 570: 7-12, 2004 